\title{
On the Dominance Solvability of Large Cournot Games*
}

\author{
TILMAN BÖRGERS
}

Department of Economics, University College London, London WCIE 6BT, United Kingdom

\author{
AND \\ Maarten C. W. Janssen \\ Department of Economics, Erasmus University Rotterdam, 3000 DR Rotterdam, \\ The Netherlands
}

Received July 20, 1992

\begin{abstract}
We consider Cournot's model of oligopolistic competition in a market for a homogeneous good. We seek conditions under which the oligopolists' game is dominance solvable and hence the Cournot equilibrium is the only outcome that survives iterated deletion of dominated strategies. We focus on "large" oligopolies, whereby we define an oligopoly to be "large" if both the demand and the supply side are replicated more than a certain finite number of times. We show that "large" Cournot oligopolies are dominance solvable if and only if the equilibrium of the approximated perfectly competitive market is globally stable under cobweb dynamics. Journal of Economic Literature Classification Numbers: 022. 1995 Academic Press, Inc.
\end{abstract}

\section{INTRODUCTION}

In perfectly competitive equilibria a high degree of coordination among market participants is achieved. The model of perfect competition does, however, not specify which strategies agents have, how the market out-

* We are grateful to an anonymous referee for suggestions that lead to substantial improvements in the paper. Tilman Börgers also thanks the "Schweizerischer Nationalfonds" for financial support. 
come depends on their strategy choices, and how agents make these choices. Instead the model resorts to the fiction of an auctioneer.

The literature on the noncooperative foundations of perfect competition tries to fill this gap. This literature has considered explicit models of oligopolistic interaction among firms. It has identified circumstances under which Nash equilibria of such models are identical, or close, to perfectly competitive equilibria. Well-known examples of oligopoly models are the Bertrand and Bertrand-Edgeworth models of price setting firms, and the Cournot model of quantity setting firms. For Bertrand and Bertrand-Edgeworth models the relation between Nash equilibria and perfectly competitive equilibria has been studied e.g. by Allen and Hellwig (1986) and Vives (1986). For Cournot models this relation has been studied e.g. by Ruffin (1971) and Novshek (1985).

For the interpretation of these results it must be born in mind that the Nash equilibrium notion implicitly assumes a substantial amount of coordination of players' behavior. Thus the results quoted, although they are more explicit about the interaction among market participants than the model of perfect competition, still assume coordination without explaining it. It is interesting to ask whether these results remain true if one replaces the notion of Nash equilibrium by a concept of strategic behavior that embodies less exogenous coordination. A particularly simple such concept is the notion of iterated deletion of dominated strategies. ${ }^{1}$

In Börgers (1992) and Janssen (1993) it has been shown that in some cases results concerning the relation between Nash equilibria of Bertrand and Bertrand-Edgeworth models and the competitive equilibrium remain true if one replaces the notion of Nash equilibrium by the notion of iterated deletion of dominated strategies. The purpose of this paper is to present a similar result for the Cournot model.

We shall consider a partial equilibrium model of a market for a homogeneous good. There is a downward sloping demand curve. The good is produced at increasing marginal costs. The number of active firms is exogenously given. We do not consider fixed costs. Firms play the standard Cournot game. In this setting Nash equilibria approximate the perfectly competitive equilibrium if the market is "large." We shall therefore focus on this case.

Two definitions of "large markets" need to be distinguished. The first definition assumes that demand is given and fixed, and that the exogenous number of firms becomes large. With this definition the aggregate competitive supply curve approaches a function that is infinitely elastic at the point in which price equals the minimum of marginal costs. Thus the

\footnotetext{
' In this paper we shall refer to iterated elimination of strongly dominated strategies. We give the precise definition of this concept in Section 4.
} 
relevant competitive equilibrium has the price equal to the minimum of marginal costs, and the equilibrium quantity equals demand at this price. The question is whether a similar outcome will emerge from strategic behaviour in a Cournot game with many firms.

If strategic behaviour is modeled using the notion of Nash equilibrium this is indeed the case (Ruffin, 1971). If, however, strategic behavior is modeled using the notion of iterated deletion of dominated strategies then a different result obtains: If the number of firms is sufficiently large all quantity choices between zero and the monopoly quantity will survive iterated deletion of dominated strategies. Increasing the number of firms thus makes the outcome of the game indeterminate. This has been shown by Bernheim (1984, pp. 1024-1025) for the Cournot model with linear demand and cost functions. A more general version of the result is due to Basu (1991, Theorem 2). ${ }^{2}$

In this paper we shall show that substantially different results are obtained if one considers an alternative definition of "large markets." This definition requires that not only the number of firms but also the number of consumers is large. We shall hence consider the case in which not only the supply side of the market, as in Bernheim (1984) and Basu (1991), but also the demand side is replicated. Under this mode of replication the competitive equilibrium is independent of the number of replications. The question is again whether the outcomes of the Cournot game approach this competitive equilibrium as the number of replications becomes large.

The answer is positive if the solution concept applied to the Cournot game is the notion of Nash equilibrium (e.g., Novshek, 1985). For the case in which the notion of strategic behaviour is iterated elimination of dominated strategies we shall show that the answer depends on the question of whether in the market under consideration the cobweb process, familiar from introductory economics textbooks, is globally stable, i.e., whether this process approaches the competitive equilibrium from every initial position.

If that is the case, then there will be a unique outcome of the Cournot game that survives iterated deletion of strongly dominated strategies, provided that the number of replications of the market exceeds some finite boundary. Trivially, this outcome will also be the unique Nash equilibrium of the Cournot game. By the result of Novshek quoted above this implies that, if the number of firms is large, the outcome surviving iterated deletion

\footnotetext{
2 Bernheim and Basu in fact use the concept of "point rationalizability" rather than the concept of "iterated deletion of dominated strategies." However, as will be shown in Section 3 , under the assumptions that we shall make, "point rationalizability" and "iterated deletion of dominated strategies" are identical concepts. In order to keep our terminology simple we therefore refer for the moment exclusively to "iterated deletion of dominated strategies."
} 
of dominated strategies is close to the competitive equilibrium. For the case that the competitive equilibrium is not globally cobweb stable we show that there is an interval of quantities that will survive iterated deletion of dominated strategies, provided again that the number of firms exceeds some finite boundary. Hence in this case the set of outcomes surviving iterated deletion of dominated strategies does not converge to the competitive equilibrium.

Our results suggest a simple intuition for Bernheim and Basu's result. With their replication method the supply function approaches a function that is infinitely elastic at the point in which price equals the minimum of marginal costs. In a market with a horizontal supply function, however, the cobweb process is obviously unstable. Thus Bernheim and Basu's indeterminacy result is analogous to our result. We emphasize that this is only an intuitive argument. Bernheim and Basu's result is not a formal implication of ours.

Our results are formulated in terms of iterated elimination of dominated strategies, but they can be rephrased as results about the dynamic stability of the Cournot equilibrium in a setting in which firms' behaviour is determined by a learning rule. Specifically, suppose that firms play the Cournot game repeatedly, and that they are "sophisticated learners" in the sense of Milgrom and Roberts (1991). We shall call the Cournot equilibrium ${ }^{3}$ "globally stable" if firms' behavior converges to the Cournot equilibrium from all initial positions. We shall show that under our assumptions for demand and cost functions the Cournot equilibrium is globally stable if and only if iterated deletion of dominated strategies leaves the Cournot equilibrium as the unique possible outcome.

This allows us to reinterpret our results as follows: If both the supply side and the demand side are replicated, then in sufficiently large markets the Cournot equilibrium will be globally stable if and only if the approximated competitive equilibrium is globally stable under the cobweb process. The same reinterpretation is possible for Bernheim and Basu's result, provided that attention is restricted to cases in which demand and cost functions satisfy the assumptions of this paper. The result then says that in a setting in which only the supply side is replicated in sufficiently large markets the Cournot equilibrium will always be unstable.

The stability of the Cournot equilibrium under simple learning rules has previously been investigated, e.g., by Theocharis (1960), Fisher (1961), and McManus and Quandt (1961). They considered only Cournot's naive learning rule, according to which each firm acts in every period assuming that all other firms behave in this period as they did in the previous period.

\footnotetext{
${ }^{3}$ We refer to "the" Cournot equilibrium because under our assumptions there will be a unique such equilibrium. See Section 3.
} 
This behaviour is allowed for in the definition of "sophisticated learning," but "sophisticated learning" encompasses many other learning rules as well. The references quoted have, moreover, restricted attention to very specific functional forms for demand and supply. Finally, these references have considered only Bernheim and Basu's mode of replication. ${ }^{4}$

Iterated deletion of dominated strategies in Cournot games has previously been investigated by Vives (1990). He applies the theory of "supermodular" games (see also Milgrom and Roberts, 1990) to Cournot games with only two firms. He shows that, under the assumptions that are also made in the current paper, Cournot games with two firms have just one outcome, namely the Cournot equilibrium, that survives iterated deletion of dominated strategies. However, the case of many firms, which is the subject of this paper, can not be dealt with using these methods.

The structure of this paper is as follows: In Section 2 we explain the intuition behind our result. In Section 3 we introduce our formal model. In Section 4 we define iterated deletion of dominated strategies, and explain why, in our model, results about iterated deletion of dominated strategies translate into results about "sophisticated learning." Our main result is then stated in Section 5. Section 6 contains some comments on the result, and in Section 7 we prove it.

\section{INTUITION}

We begin with some preliminary considerations. They will be stated more rigorously in Section 4 . In the previous section we began by explaining our result as a result concerning iterated deletion of dominated strategies. In our model this will, however, be the same as iterated deletion of strategies that are not best replies to pure strategy combinations. ${ }^{5}$ Strategies that are left over after this latter procedure have been called "point rationalizable" (Bernheim, 1984).

The equivalence between iterated deletion of dominated strategies and point rationalizability follows from a result of Moulin (1984). Moulin's result basically says that the two concepts are equivalent in games in which players' strategy sets are one-dimensional intervals, and in which

\footnotetext{
${ }^{4}$ Some papers have considered learning rules for Cournot games which are not encompassed by "sophisticated learning." They include Fisher (1961), Hahn (1962), Al-Nowaihi and Levine (1985), and Thorlund-Petersen (1990).

${ }^{s}$ It is important that we refer here only to combinations of pure, not of mixed, strategies. This is because in general games (though not in our model) iterated deletion of strategies that are not best replies to any combination of pure strategies can be a substantially more restrictive concept than iterated deletion of strategies that are not best replies to any combination of mixed strategies. See Bernheim (1984).
} 
their payoffs are strictly quasi-concave. Our model will yield obvious bounds for the firms' choices of quantities, and hence the first condition will be satisfied. To ensure that the second condition also holds we shall make appropriate assumptions for demand and cost functions.

Our main result can hence also be viewed as providing conditions under which large Cournot games have just one point-rationalizable strategy for each firm. This, in turn, is easily shown to be equivalent to the statement that the Cournot equilibrium is globally stable under Cournot's naive learning rule. We have thus obtained apparently more special, but in fact equivalent, verisons both of our basic result and of its reinterpretation as a result about learning stability. It is easier to explain the intuition for the simpler versions of our results.

Consider a market with a continuum of both firms and consumers. Suppose that firms play the Cournot game repeatedly, and that they chose their strategies using Cournot's naive learning rule. Since there is a continuum of firms each firm will regard the market price as independent of its own decisions. Hence, in any period, every firm will expect the previous period's price to continue to prevail also in the current period. Moreover, its quantity choice will be its competitive supply. Thus its behavior will be the one described by the cobweb model. This equivalence of the Cournot and the cobweb process in markets with continua of agents makes it trivial that in such markets the Cournot process is globally stable if and only if the cobweb process is globally stable. ${ }^{6}$ Guesnerie (1992) has made the closely related observation that in a Cournot game with continua of agents there will be a unique point rationalizable strategy if and only if the cobweb process is globally stable. ${ }^{7}$

The above observations for models with continua of agents can be regarded as results "in the limit." In this paper we present corresponding results for finite markets. Our results are (perhaps) surprisingly strong in that they show that what is true in the limit is also exactly (not only approximately) true in finite, sufficiently large markets. To obtain these results we shall assume differentiability of demand and cost functions, and we shall impose regularity conditions "in the limit." We shall introduce a sense in which these conditions will "generically," but not always, be satisfied. They will make the properties of the limit markets robust under perturbations.

\footnotetext{
${ }^{6}$ This equivalence has previously been observed by Fisher (1961). However, he considers a market with a finite number of firms, and assumes that firms (mistakenly) consider themselves, and act, as price-takers.

${ }^{7}$ Guesnerie does not interpret his model as a Cournot game. However, it is obvious that such an interpretation of his model is possible.
} 


\section{THE MOdeL}

We consider the market for a homogeneous good. The demand function is $D: \mathbb{R}_{+} \rightarrow \mathbb{R}_{+}$. For any price $p \in \mathbb{R}, D(p)$ is the quantity demanded at price $p$. We assume that there is some $\bar{p}>0$ such that $D(p)>0$ if and only if $p \in[0, \bar{p})$. We also assume that $D$ is twice continuously differentiable on $[0, \bar{p}],{ }^{8}$ and that $D^{\prime}(p)<0$ for all $p \in[0, \bar{p}]$. This implies that $D$ is strictly decreasing on this interval. We can then define the inverse demand $P: \mathbb{R}_{+} \rightarrow \mathbb{R}_{+}$by setting $P(0) \equiv \bar{p}, P(q) \equiv D^{-1}(q)$ if $q \in(0, D(0)]$, and $P(q) \equiv 0$ if $q>D(0)$. Our differentiability assumption for $D$ implies that $P$ is twice continuously differentiable on $[0, D(0)]$.

We assume that $P^{\prime}(q)+P^{\prime \prime}(q) q<0$ for all $q \in[0, D(0)]$. This assumption which was first introduced by Hahn (1962) has been used in many treatments of the Cournot model. With downward sloping demand Hahn's condition implies, but is more restrictive than, the requirement that revenue is concave. On the other hand, Hahn's condition is less restrictive than the requirement that inverse demand is concave. Together with the convexity of costs (see below), the assumption ensures that the Cournot oligopolists' profit functions are strictly quasi-concave in their own quantity choice, and, moreover, that their profit maximizing quantity choices are a decreasing function of the total supply of all other firms.

Hahn's condition is rather restrictive. We conjecture, however, that our results remain true if Hahn's condition is violated. This is because our results refer to "large" markets, and in large markets the implications of Hahn's condition that we make use of can be derived from mild regularity assumptions only. ${ }^{9}$ Giving up Hahn's assumption would, however, make our arguments more cumbersome, and therefore we maintain the assumption in this paper.

The costs at which the good that we consider can be produced are described by a cost function $C: \mathbb{R}_{+} \rightarrow \mathbb{R}_{+}$. For any $q \in \mathbb{R}_{+}, C(q)$ are the costs of producing $q$. We assume that $C$ is twice continuously differentiable on $\mathbb{R}_{+}$, that $C^{\prime}(q) \geq 0$ and that $C^{\prime \prime}(q)>0$ for all $q \in \mathbb{R}_{+}$. Costs are hence strictly increasing and strictly convex. We can now define the supply

\footnotetext{
${ }^{8}$ By continuous differentiability of $D$ on $[0, \bar{p}]$ we mean that $D$ is differentiable on the interior of $[0, \bar{p}]$, that in 0 (resp., $\bar{p}$ ) the right hand (resp., left hand) derivative of $D$ exists as well, and that these derivatives are continuous on $[0, \bar{p}]$. $D$ is twice continuously differentiable on $[0, \bar{p}]$ if the above statements also apply to the derivative of $D$ on this interval. Observe that this allows for the possibility that $D$ is not differentiable in $\bar{p}$, if it is regarded as a function defined on the whole of $\mathbb{R}_{+}$. We shall use analogous terminology throughout this paper, also in the context of functions other than $D$.

${ }^{9}$ This is a familiar point in the literature on large Cournot markets; see, for example, the proof of part (i) of the "Theorem" in Novshek (1985). In our context the point can be understood through inspection of the derivatives calculated in Lemma 4 in Section 7.
} 
function $S: \mathbb{R}_{+} \rightarrow \mathbb{R}_{+}$by setting $S(p) \equiv 0$ if $p \in\left[0, C^{\prime}(0)\right]$, and $S(p) \equiv$ $\left(C^{\prime}\right)^{-1}(p)$ if $p>C^{\prime}(0)$. To exclude trivial cases we assume $C^{\prime}(0)<\bar{p}$.

Starting from these basic functions we shall now consider oligopolistic markets. We shall replicate both the demand side and the supply side. If the number of replications is $N(\geq 2)$ we shall speak of the " $N$ th Cournot game." In the $N$ th Cournot game, demand is $D^{N}(p)=N D(p)$ for every $p \in \mathbb{R}_{+}$. Inverse demand is therefore $P^{N}(0) \equiv \bar{p}, P^{N}(Q) \equiv\left(D^{N}\right)^{-1}(Q)$ if $Q \in(0, N D(0)]$, and $P^{N}(Q) \equiv 0$ if $Q>N D(0)$. Observe that $P^{N}(Q)=$ $P(Q / N)$ for all $Q \in \mathbb{R}_{+}$. We shall use this relation. In the $N$ th Cournot game there are $N$ firms. They all have the same cost function, $C$.

The specification of the game itself is standard: Every firm $i \in\{1,2$, $\ldots, N\}$ chooses the quantity $q_{i} \in \mathbb{R}_{+}$that it produces. Choices are made simultaneously and independently. The market price is $P\left((1 / N) \sum_{i=1}^{N} q_{i}\right)$. Each firm $i$ seeks to maximize its profits: $q_{i} P\left((1 / N) \sum_{j=1}^{N} q_{j}\right)-C\left(q_{i}\right)$.

Observe that we restrict attention to symmetric Cournot games. This is however only to simplify the presentation. Our results could easily be adapted to the case that there is a finite collection of possible cost functions, and that for every possible cost function there are $N$ firms producing with this cost function.

A Nash equilibrium of a Cournot game is a vector of quantities, one for each firm, such that each firm maximizes its profit, taking the other firms' quantities as given and fixed. Nash equilibria of Cournot games will also be called "Cournot equilibria." As Theorem 1 in Friedman (1982) shows, our assumptions imply that for any number $N$ of firms there is a unique Cournot equilibrium. In this equilibrium all firms produce the same quantity. We shall denote this quantity by $q^{N . C}$.

For our further discussions we also need to refer to the competitive (Walrasian) equilibrium. The competitive price $p^{w}$ is defined by $S\left(p^{W}\right)=$ $D\left(p^{W}\right)$. Note that $p^{W}$ is unique. The corresponding quantity is $q^{W}=S\left(p^{W}\right)$. Observe that for $N \rightarrow \infty$ we have $q^{N . C} \rightarrow q^{W}$. This is shown in Novshek $(1985) .^{10}$

\section{Solution Concepts}

We plan to solve Cournot games using iterated deletion of dominated strategies. The notion of dominance that we shall be using is "strong dominance." A strategy of a firm $i$ is "strongly dominated" if there is another strategy of firm $i$ that gives firm $i$ strictly higher profit independent

\footnotetext{
${ }^{10}$ Novshek assumes that average costs are bounded away from zero. We have not made such an assumption. However, in our framework convergence of Cournot equilibrium holds also in the absence of this assumption. This can e.g. easily be derived from Lemma 7 in Section 7 below.
} 
of what all other firms do. Iterated deletion of strongly dominated strategies is then the following procedure: We first delete from the original game all strategies that are strongly dominated. Then we delete from the resulting, reduced game all strategies that are strongly dominated in this reduced game. We obtain a further reduced game. In this game we again eliminate all strongly dominated strategies, etc. A strategy is said to "survive iterated deletion of strongly dominated strategies for firm $i$ " if after any finite number of steps it is still contained in firm $i$ 's strategy set.

As was mentioned in Section 2, a result of Moulin (Lemma 2 in Moulin, 1984) implies that in our model iterated deletion of dominated strategies is equivalent to iterated deletion of strategies that are not best replies to pure strategy combinations. "Moulin's result concerns games in which the players' strategy sets are closed intervals of real numbers, and in which their payoffs are continuous and strictly quasi-concave. In our context, the first condition is not problematic, since, obviously, firms will never wish to choose a quantity above $N D(0)$. The other conditions follow immediately from the assumptions of Section 3.

As Moulin's result is crucial for what follows we briefly explain the logic behind it. The important part of the argument for the asserted equivalence shows that at each stage of the iterated deletion of strategies that are not best replies all eliminated strategies are also strongly dominated. Now, observe first that if we start with closed intervals as players' strategy sets, then uniqueness and continuity of best replies imply that at each stage the set of best replies of any player is still a closed interval. Therefore, it is sufficient to show that every strategy that is larger than the largest best reply, or smaller than the smallest best reply, is strongly dominated. But strict quasi-concavity implies that whatever the other players choose, any player's payoff will be monotonically decreasing to the right of his largest best reply. Hence, the largest best reply strongly dominates all larger strategies. Similarly, the smallest best reply strongly dominates all smaller strategies.

We continue with a formal definition of iterated deletion of strategies that are not best replies to pure strategy combinations. We first define the firms' best reply functions. Consider some firm $i$ in the $N$ th Cournot game. Suppose all other firms had decided about their production quantities. Suppose that the total quantity produced by the other firms were $(N-1) q_{-i}$, so that the average quantity produced by the other firms were $q_{-i} \cdot{ }^{12}$ Firm $i$ will then choose its own quantity $q_{i}$ so as to maximize its

\footnotetext{
"Moulin refers to iterated deletion of weakly dominated strategies, not, as we do, to iterated deletion of strongly dominated strategies. However, his proof applies without change to our case as well.

${ }_{12}$ Observe that $q_{-i}$ stands for the average of the quantities produced by all firms other than firm $i$, not for the vector of these quantities, as would be the more conventional notation.
} 
profits $P\left(((N-1) / N) q_{-i}+(1 / N) q_{i}\right) q_{i}-C\left(q_{i}\right)$. Our assumptions imply that for every $q_{-i} \in \mathbb{R}_{+}$firm $i$ 's profits are a strictly quasi-concave function of its own choice $q_{i}$. Hence firm $i$ 's profit maximization problem has a unique solution. We denote this solution by $B^{N}\left(q_{-i}\right)$. We do not give this expression an index $i$ because it obviously does not depend on $i$.

We observe that $B^{N}$ is continuous, that there is some $\bar{q}_{-i}^{N}>0$ such that $B^{N}\left(q_{-i}^{N}\right)>0$ if and only if $q_{-i} \in\left[0, \bar{q}_{-i}^{N}\right)$, and that $B^{N}$ is strictly decreasing on $\left[0, \bar{q}_{-i}^{N}\right]$. The proofs of these facts are standard. The assertions are also implied by Lemma 4 in Section 7.

We can now define iterated deletion of strategies that are not best replies. Let again $N$ be given. Let

$$
R^{N, 0} \equiv \mathbb{R}_{+}
$$

and for any $n \in \mathbb{N}$ define

$$
R^{N, n} \equiv\left\{q_{i} \in R^{N, n-1} \mid q_{i}=B^{N}\left(q_{-i}\right) \text { for some } q_{-i} \in R^{N, n-1}\right\} .
$$

Finally, define

$$
R^{N} \equiv \bigcap_{n \in \mathbb{N}} R^{N, n}
$$

(Here $i$ is any arbitrary firm. We have not given the sets $R^{N . n}$ and $R^{N}$ an index $i$ because they obviously do not depend on $i$.)

Let us verify that this procedure is indeed iterated deletion of strategies that are not best replies to pure strategy combinations. To begin with, it is clear that in the first step a strategy is eliminated if and only if it is not a best reply to any pure strategy combination of the other players. To see that the same also holds for all further steps, note first that an inductive argument can be used to show that for every $n \in \mathbb{N}$ the set $R^{N, n}$ is a finite, closed interval. Therefore, if all firms except firm $i$ choose a quantity in $R^{N, n}$ then also the average of these quantities will be in $R^{N, n}$. Hence firm $i$ 's best reply must be in $R^{N, n+1}$. On the other hand, of course, all strategies that are contained in $R^{N, n+1}$ are best replies to a strategy combination of the other firms in which all these firms choose pure strategies in $R^{N, n}$. Hence in the game with strategy sets $R^{N, n}$ the set of best replies to pure strategy combinations for any firm $i$ is precisely $R^{N, n+1}$, and hence the procedure described above is just iterated deletion of strategies that are not best responses to pure strategy combinations.

Note that for every $N \geq 2$ the set $R^{N}$ is a nonempty, closed, and bounded 
interval. This follows from the fact that $R^{N}$ is defined as the intersection of a nested sequence of such intervals.

We next come to the claim made in Section 2 that the sets $R^{N}$ are singletons if and only if Cournot's learning process is globally stable. We call a sequence $\left\{\left(q_{1}^{n}, q_{2}^{n}, \ldots, q_{N}^{n}\right)\right\}_{n \in \mathbb{N}}$ a "Cournot sequence" if and only if $q_{i}^{n}=B^{N}\left(1 /(N-1) \sum_{j \neq i} q_{j}^{n-1}\right)$ for all $n \in \mathbb{N}$ with $n \geq 2$, and for all $i$. Observe that the continuity of the best reply function $B^{N}$ implies that every convergent Cournot sequence must converge to the Cournot equilibrium. We shall say that the Cournot process is globally stable if and only if all Cournot sequences are convergent.

The fact that $\# R^{N}=1$ implies that the Cournot process is globally stable is well-known from Moulin (1984, Theorem 1). ${ }^{13}$ To see the converse suppose that $R^{N}$ were an interval with nonempty interior. Suppose that in period 1 all firms produced $\min R^{N}$, in period 2 all firms produced max $R^{N}$, in period 3 all firms produced again $\min R^{N}$, etc. The monotonicity of the best reply function $B^{N}$ implies that this would constitute a Cournot sequence. Obviously this sequence does not converge. Hence the Cournot process would not be globally stable. This establishes the asserted equivalence.

Finally, we have claimed above that it is also true that the strategy sets surviving iterated deletion of strongly dominated strategies, and hence the sets $R^{N}$, are-singletons if and only if "sophisticated learning" in the sense of Milgrom and Roberts (1991) $)^{14}$ is globally stable. We shall not give a formal statement and proof of our assertion. We only note that the "only if" part can be shown as in Theorem 5 of Milgrom and Roberts (1991), and that the "if" part follows from the argument that was used in the analogous context in the preceding paragraph.

\section{LARGE MARKETS}

We now consider properties of the sets $R^{N}$ in the case that $N$ is large. As was explained in the Introduction the results that we wish to present depend on properties of the cobweb process in our model. We therefore first study some properties of this process. In the cobweb process it is assumed that firms behave as "price-takers." In each period they produce their competitive supply, given last period's price. This period's price then adjusts so as to clear the market. Formally, a sequence $\left\{q^{n}\right\}_{n \in \mathbb{N}}$ of

\footnotetext{
${ }^{13}$ Footnote 11 applies to this result of Moulin as well.

${ }^{14}$ Whereby, however, in Milgrom and Roberts' definition (Milgrom and Roberts, 1991, p. 89) we set $\varepsilon=0$ (using their notation). Allowing for strictly positive $\varepsilon$ is in our context an irrelevant complication.
} 
elements of $\mathbb{R}_{+}$is called a "cobweb sequence" if $q^{n}=S\left(P\left(q^{n-1}\right)\right)$ for all $n \in \mathbb{N}$ with $n \geq 2$.

Observe that for any cobweb sequence $q^{1} \leq q^{w}$ implies that $q^{n} \leq q^{w}$ for all odd $n$, and $q^{n} \geq q^{w}$ for all even $n$. Analogously, $q^{1} \geq q^{w}$ implies that $q^{n} \geq q^{w}$ for all odd $n$, and $q^{n} \leq q^{w}$ for all even $n$. Hence every cobweb sequence can be partitioned into two subsequences, one consisting of all odd-numbered elements of the sequence, the other consisting of all even-numbered elements of the sequence. One of these subsequences will stay above the competitive quantity, the other one will stay below the competitive quantity (in both cases equality is not excluded). Since we shall only be interested in the long run behaviour of cobweb sequences there is no loss of generality in restricting attention to the case that it is the odd-numbered sequence that stays above the competitive quantity. We shall make this assumption throughout.

Observe next that cobweb sequences are bounded from below by zero, and from above by $\max \left\{S(\bar{p}), q^{1}\right\}$. Note also that $q^{1} \leq q^{3}$ implies that $q^{3} \leq q^{5} \leq q^{7} \leq \cdots$, and $q^{2} \geq q^{4} \geq q^{6} \geq \cdots$. Analogously, $q^{1} \geq q^{3}$ implies that $q^{3} \geq q^{5} \geq q^{7} \geq \cdots$ and $q^{2} \leq q^{4} \leq q^{6} \leq \cdots$. Thus both the odd and the even numbered sequences are bounded and monotonic. Hence they must be convergent. Let us denote by $\bar{q}$ the limit of the odd-numbered sequence, and let us denote by $q$ the limit of the even-numbered sequence. By the continuity of the functions involved we have $\bar{q}=S(P(q))$ and $q=S(P(\bar{q}))$. Thus, either $\bar{q}=q=q^{W}$, in which case the cobweb sequence itself also converges to the competitive quantity, or $q<q^{w}<\bar{q}$. In the latter case the sequence $\bar{q}, \underline{q}, \bar{q}, \underline{q}, \ldots$ itself is a cobweb sequence. We shall then call the pair $(\bar{q}, q)$ a "cobweb cycle," and we shall say that the cobweb sequence "converges to the cobweb cycle $(\bar{q}, \underline{q})$."

Now suppose that the demand function $D$ and the cost function $C$ are given and fixed. If all cobweb sequences converge to the competitive quantity $q^{w}$ we shall say that "the cobweb process is globally stable." Otherwise we shall call it "unstable." Our purpose is to present two results concerning the properties of the sets $R^{N}$ for large $N$. One result will concern the case in which the cobweb process is globally stable, the other will concern the case in which it is unstable. Both results will be built on a small extra assumption which is generically, but not always, satisfied. If these extra assumptions are satisfied we shall say that "the cobweb process is strictly globally stable," resp. that "the cobweb process is strictly unstable."

We now give the precise definitions of these terms. The cobweb process is strictly globally stable if it is globally stable and if, in addition, in the competitive equilibrium the slope of supply is in absolute terms smaller than the slope of demand, i.e., if

$$
S^{\prime}\left(p^{w}\right)<\mid\left(D^{\prime}\left(p^{w}\right) \mid\right.
$$


Intuitively, this condition says that the cobweb process would remain globally stable if supply and demand were replaced by their local linear approximations, whereby "local" refers to the neighborhood of the competitive equilibrium.

The cobweb process is strictly unstable if it is unstable and if, in addition, there exists a cobweb sequence $q^{1}, q^{2}, q^{3}, \ldots$ with $q^{1}<q^{3}$. Hence there must exist at least one cobweb sequence for which the sequence above $q^{W}$ is initially strictly increasing. Note that what we have said earlier implies that such a sequence cannot exist if the cobweb process is globally stable.

The role which these conditions play will become clear in Section 7 when we prove our result. It was claimed above that these conditions will "generically" be satisfied. We shall give an argument for this claim in Section 6. Our result now is the following.

Proposition. (i) If the cobweb process is strictly globally stable then there exists a $\bar{N} \in \mathbb{N}$ such that $N \geq \bar{N}$ implies

$$
R^{N}=\left\{q^{N, C}\right\}
$$

(ii) If the cobweb process is strictly unstable then there exist a $\bar{N} \in \mathbb{N}$ and an interval $\mid \hat{q}, \hat{q}] \subseteq \mathbb{R}_{+}$such that $q^{w}$ is contained in the interior of $[\hat{q}, \hat{q}]$ and such that $N \geq \bar{N}$ implies

$$
[\hat{q}, \hat{q}] \subseteq R^{N} .
$$

Observe that the convergence property of Cournot equilibria, if combined with part (i) of the proposition, shows that if the cobweb process is strictly stable then the sets $R^{N}$ converge (for $N \rightarrow \infty$ ) in the Hausdorff topology to the singleton $\left\{q^{w}\right\}$. On the other hand, part (ii) shows, obviously, that if the cobweb process is strictly unstable the sets $R^{N}$ will not Hausdorff-converge to the set $\left\{q^{w}\right\}$.

The proposition is phrased as a result about iterated deletion of strongly dominated strategies. But, as explained in Section 4, it could equivalently be formulated as a result about the stability of the Cournot equilibrium under "sophisticated learning." Moreover, as explained in Section 2, the intuition for the proposition is derived from the fact that in large markets sophisticated learning in the Cournot model is a dynamic process that is similar to the cobweb process. Thus, yet another way of phrasing our main result would be to state it as a result about the robustness of the convergence properties of a certain dynamic system under small perturbations.

This last reformulation of our result is in fact independent of the eco- 
nomic interpretation of the dynamic process involved, and depends only on the fact that the cobweb process is a dynamic process with a Markov structure, i.e., a memory of just one period, with state space $\mathbb{R}_{+}$, and with a monotonically decreasing transition function. That these properties suffice will become clear from the proof of the result.

We shall prove the proposition in Section 7. In Section 6 we first discuss the role of the "strictness" assumptions in the proposition. We then also explain in which sense the "strictness" assumptions in the proposition are "generically" satisfied.

\section{Discussion}

In this section we first discuss how far the "strictness" assumptions in the proposition can be relaxed. It will turn out to be helpful to combine the discussion of this question with a discussion of the question of whether the differentiability assumptions can be relaxed.

As stated, part (i) of the Proposition asserts that for large $N$ the sets $R^{N}$ will be singletons provided that the cobweb process is strictly stable. Our proof of this part of the proposition, as it will be presented in the next section, makes essential use of both the strictness assumption and of differentiability. We cannot extend it to the case in which these assumptions are violated.

However, as we mentioned in the previous section, an implication of the conclusion of part (i) of the proposition is the Hausdorff convergence of the sets $R^{N}$ to the set $\left\{q^{W}\right\}$. We can prove this weaker result also if the strictness assumption is relaxed and only stability of the cobweb process is assumed. The proof is a straightforward application of results that will be presented in Section 7. We omit the details.

If one is only interested in Hausdorff convergence of the sets $R^{N}$ we can in fact go one step further and relax not only the strictness assumption but (at the same time) also the differentiability assumptions. We do need, however, the assumptions that we expressed in terms of derivatives, i.e., strict quasi-concavity of profits, and continuity and monotonicity of best replies. Again, the argument for this is straightforward from Section 7 , and is therefore omitted.

As regards part (ii) of the proposition we observe first that this result remains true without any qualifications if differentiability is relaxed. The proof that we give below applies immediately also to this case.

We next ask whether the strictness assumption in part (ii) can also be relaxed without affecting the conclusion, or whether at least, if strictness does not hold, it will remain true that the sets $R^{N}$ do not converge (for $N \rightarrow \infty$ ) in the Hausdorff topology to the set $\left\{q^{W}\right\}$. 
To investigate this question we have considered numerical examples in which the cobweb process is unstable, but not strictly unstable. For some of these examples we found that the sets $R^{N}$ did not converge (for $N \rightarrow$ $\infty)$ to the set $\left\{q^{W}\right\}$. For other examples, however, convergence did obtain. Hence the conclusion of part (ii) of the proposition did not generalize. In order to simplify calculations we considered examples in which the supply functions are piecewise linear. Hence our examples violated both the differentiability assumptions and the strictness assumption. We conjecture, however, that the case in which only the strictness assumption is violated is not different.

In the rest of this section we wish to argue that the "strictness" assumptions in the proposition will "generically" be satisfied. This will be intuitively plausible for the case of stability, but for the case of instability it may not be obvious. It is therefore mainly for this case that we include the following discussion.

We first make precise the sense in which we use the expression "generically." Let $\mathcal{M}^{1}$ (resp. $\mathcal{M}^{2}$ ) denote all those pairs $(D, C)$ of a demand and a cost function that satisfy the assumptions of Section 3 and for which the cobweb process is globally stable (resp., unstable). Let $\bar{M}^{1}$ (resp., $\bar{M}^{2}$ ) denote all those pairs in $\mathcal{M}^{1}$ (resp., $\mathcal{M}^{2}$ ) for which the cobweb process is strictly globally stable (resp., strictly unstable). We shall define "genericity" topologically. Hence our assertion will be that $\bar{M}^{1}$ (resp., $\bar{M}^{2}$ ) is an open and dense subset of $\mathcal{M}^{\prime}$ (resp., $M^{2}$ ).

To give these claims meaning we need to endow the sets $\mu^{1}$ and $M^{2}$ with a topology. For this we first define separate topologies for demand functions and for cost functions. The sets $M^{1}$ and $\mathcal{M}^{2}$ are then endowed with the relative topologies induced by the product of these two topologies.

Intuitively we shall endow the set of all demand functions with the topology of uniform $C^{\prime}$-convergence, and we shall endow the set of all cost functions with a topology which induces the topology of uniform $C^{-1}$-convergence for supply functions. It is important that our topologies refer not only to the demand and supply functions themselves, but also to their first derivatives. The stability of a dynamic process such as the cobweb process depends not only on the values of the relevant functions, but also on their derivatives. In fact, one can easily show that if we referred only to the demand and supply functions themselves, and not to their first derivatives, among all pairs of demand and supply functions those for which the cobweb process is unstable would be an open and dense subset. Hence stability itself would be nongeneric. This would not be a useful notion of "genericity" for our purposes. With the topology to be introduced below this problem does not arise.

We now sketch the formal definition of our topologies. As regards demand functions, we have to take account of the possible nondifferenti- 
ability of demand functions in the point in which they cut the quantity axis. We therefore define that two demand functions are "close" if and only if their supports are close in the Hausdorff topology (whereby we define the "support" of a demand function to be the set of all prices for which demand is strictly positive) and if, on the intersection of their supports, both the functions themselves and their first derivatives are close in terms of the uniform topology. The topology for cost functions is simply the topology of uniform $C^{2}$-convergence, i.e., the topology of uniform convergence of the functions themselves and of their first and second derivatives.

The fact that, with these definitions, $\bar{M}^{1}$ is an open and dense subset of $\mu^{1}$ is obvious. As regards $\bar{M}^{2}$, the fact that this set is open in $M^{2}$ will also be obvious. It remains to be shown that $\bar{M}^{2}$ is dense in $\mathcal{M}^{2}$. So let $(D, C) \in \mathcal{M}^{2}$. We have to show that we can find arbitrarily small perturbations of $(D, C)$ that are contained in $\bar{M}^{2}$.

Observe first that our earlier discussion shows that there must be a cobweb cycle $(\bar{q}, q)$ for $(D, C)$. Now suppose that we left demand unchanged, and that we adjusted costs so that supply at prices below the competitive price remained unchanged, but supply at prices above the competitive price slightly increased. Clearly such a pair of demand and cost functions can be found, and it can be made arbitrarily close to $(D$, $C$ ) in the above topology. We will establish our claim by showing that such a pair will be in $\bar{M}^{2}$. For this we have to show that a cobweb sequence $q^{1}, q^{2}, q^{3}, \ldots$ with $q^{3}>q^{1}$ exists. Now set $q^{1}=\bar{q}$. Then by construction $q^{2}=\underline{q}$. Now, with the old supply function, $S(P(q))=\bar{q}$. But in our construction we have increased supply at prices such as $P(q)$. Denoting the new supply function by $\bar{S}$ we now have $q^{3}=\tilde{S}(P(q))>\overline{\bar{q}}=q^{1}$. This completes our argument.

\section{ProOF}

We proceed in a sequence of lemmas. In Lemmas $1-3$ we will collect some properties of the function $S(P()$.$) and its twofold iterate. These$ functions govern the cobweb process. In Lemmas $4-6$ we shall describe analogous properties of the functions $B^{N}$ (for $N \in \mathbb{N}$ with $N \geq 2$ ) and their twofold iterates. These functions determine the sets of strategies that survive iterated deletion of strongly dominated strategies in the Cournot games. Lemmas 7 and 8 concern the relation between the functions $B^{N}$ and the function $S(P()$.$) and the relation between the twofold iterates of$ these functions, in both cases considering the case that $N$ grows large. We conclude the section by combining the lemmas to provide the proof 
of the proposition. Most of the lemmas are elementary, and therefore we give no formal proof for them.

We begin by considering the function $S(P()$.$) . We simplify notation and$ write $F($.$) for S(P()$.$) .$

LEMma 1. (i) $F(q)>0$ if and only if $q \in\left[0, D\left(C^{\prime}(0)\right)\right)$.

(ii) $F$ is continuously differentiable on $\left[0, D\left(C^{\prime}(0)\right)\right]$. Its derivative satisfies

$$
F^{\prime}(q)=\frac{P^{\prime}(q)}{C^{\prime \prime}(F(q))} \quad(<0)
$$

for all $q \in\left[0, D\left(C^{\prime}(0)\right)\right]$.

As was explained in the Section 5 every cobweb sequence converges either to the competitive equilibrium quantity or to a cycle of period 2 . It is therefore useful to consider the twofold iterate of $F$ which we shall denote by $\tilde{F}: \bar{F}(q) \equiv F(F(q))$ for all $q \in \mathbb{R}_{+}$.

LEMMA 2. (i). If $S(\bar{p})>D\left(C^{\prime}(0)\right)$ then there are $y, z>0$ with $y<z$ such that $\tilde{F}(q)=0$ if and only if $q \in[0, y]$, and $\tilde{F}(q)=S(\bar{p})$ if and only if $q \geq z$. Moreover, on $[y, z] \bar{F}$ is continuously differentiable. Its derivative satisfies $\tilde{F}^{\prime}(q)=F^{\prime}(q) F^{\prime}(F(q))(>0)$ for all $q \in[y, z]$.

(ii) If $S(\bar{p}) \leq D\left(C^{\prime}(0)\right)$, then there is some $z>0$ such that $\tilde{F}(q)=$ $S(\bar{p})$ if and only if $q \geq z$. Moreover, on $[0, z] \tilde{F}$ is continuously differentiable. Its derivative satisfies $\tilde{F}^{\prime}(q)=F^{\prime}(q) F^{\prime}(F(q))(>0)$ for all $q \in[0, z]$.

The next lemma describes the relevance of fixed points of $\tilde{F}$ :

LEMMA 3. (i) $q^{w}$ is a fixed point of $\bar{F}$.

(ii) For all $q \neq q^{w}: q$ is a fixed point of $\tilde{F}$ if and only if there is a cobweb cycle $(\bar{q}, \underline{q})$ and $q \in\{\bar{q}, \underline{q}\}$.

We conclude our discussion of the function $\bar{F}$ with the following observations which combine parts of our discussion in Section 5 with parts of the above lemmas. If the cobweb process is globally stable, then $q^{w}$ is the unique fixed point of $\bar{F}$. If the cobweb process is strictly globally stable, then $q^{w}$ is the only fixed point of $\bar{F}$, and, moreover, $\tilde{F}^{\prime}\left(q^{w}\right)<1$. If the cobweb process is unstable, then $\tilde{F}$ must have fixed points that are smaller than $q^{w}$, and fixed points that are larger than $q^{W}$. If the cobweb process 


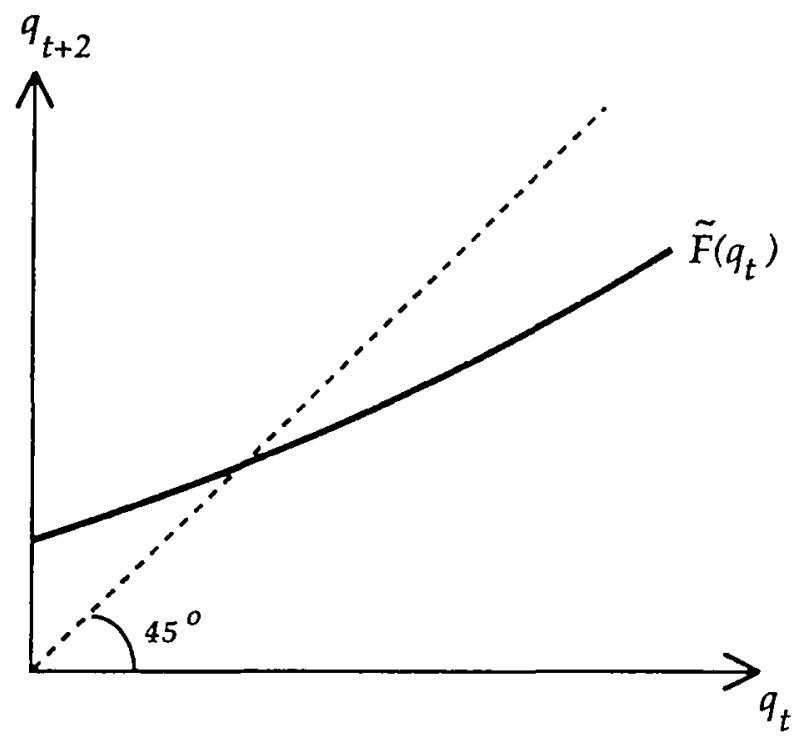

FIG. 1. Strictly stable cobweb process.

is strictly unstable then $\tilde{F}(q)<q$ for some $q \in\left(0, q^{w}\right)$. In Figs. 1-4 we show the "typical" shape of the graph of $\tilde{F}$ for each of these four cases. These figures are intended to provide a geometric aid for reading the remainder of the proof.

Just as the cobweb process is governed by the function $F$ and its twofold iterate $\bar{F}$, similarly, for every $N \in \mathbb{N}$ with $N \geq 2$, the sets of strategies that survive iterated deletion of strongly dominated strategies are determined by the function $B^{N}$ and its twofold iterate. We shall write $\bar{B}^{N}$ for the twofold iterate. The properties of these functions are similar to the properties of the functions $F$ and $\tilde{F}$. We begin by stating the analogue of Lemma 1.

Lemma 4. For all $N \in \mathbb{N}$ with $N \geq 2$,

(i) $B^{N}\left(q_{-i}\right)>0$ if and only if $q_{-i} \in\left[0,(N /(N-1)) D\left(C^{\prime}(0)\right)\right)$.

(ii) $B^{N}$ is continuously differentiable on $\left[0,(N /(N-1)) D\left(C^{\prime}(0)\right)\right]$. Its derivative satisfies

$$
B^{N^{\prime}}\left(q_{-i}\right)=\frac{X}{Y} \quad(<0)
$$

whereby 


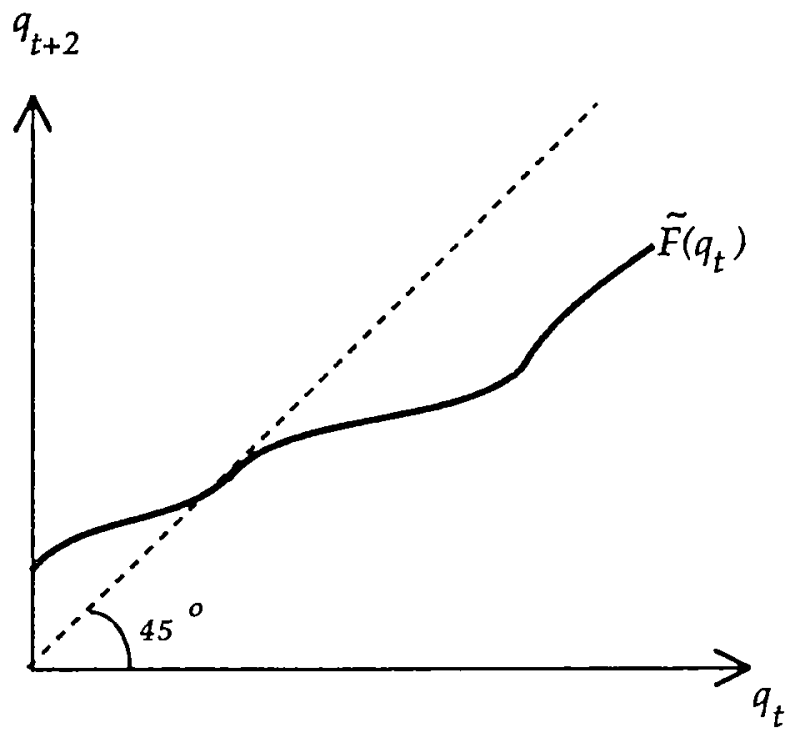

FIG. 2. Stable, but not strictly stable, cobweb process.

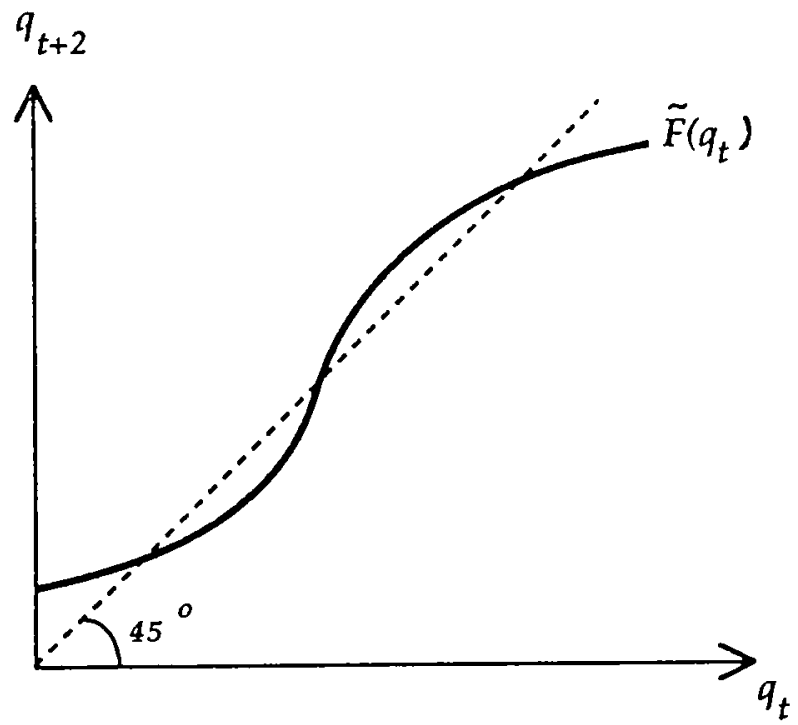

FIG. 3. Strictly unstable cobweb process. 


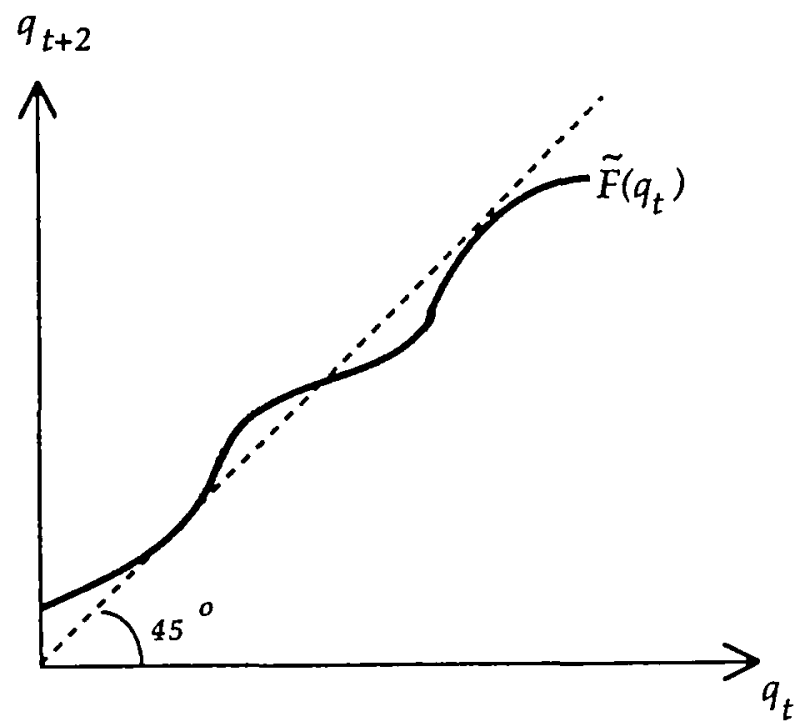

Fig. 4. Unstable, but not strictly unstable, cobweb process.

$$
\begin{aligned}
X \equiv & \frac{N-1}{N} P^{\prime}\left(\frac{N-1}{N} q_{-i}+\frac{1}{N} B^{N}\left(q_{-i}\right)\right) \\
& +\frac{N-1}{N^{2}} P^{\prime \prime}\left(\frac{N-1}{N} q_{-i}+\frac{1}{N} B^{N}\left(q_{-i}\right)\right) B^{N}\left(q_{-i}\right)
\end{aligned}
$$

and

$$
\begin{aligned}
Y \equiv & C^{\prime \prime}\left(B^{N}\left(q_{-i}\right)\right)-\frac{2}{N} P^{\prime}\left(\frac{N-1}{N} q_{-i}+\frac{1}{N} B^{N}\left(q_{-i}\right)\right) \\
& -\frac{1}{N^{2}} P^{\prime \prime}\left(\frac{N-1}{N} q_{-i}+\frac{1}{N} B^{N}\left(q_{-i}\right)\right) B^{N}\left(q_{-i}\right) .
\end{aligned}
$$

To understand this lemma observe that $q_{-i} \in\left[0,(N /(N-1)) D\left(C^{\prime}(0)\right)\right)$ means that the total quantity produced by all firms other than $i,(N-$ 1) $q_{-i}$, is in $\left[0, D^{N}\left(C^{\prime}(0)\right)\right)$, and hence that if firm $i$ chooses to produce an output close to zero, the resulting price will exceed marginal costs at zero. Therefore, in this interval, $B^{N}$ will be positive.

The differentiability of $B^{N}$ on $\left[0,(N /(N-1)) D\left(C^{\prime}(0)\right)\right]$ follows from the implicit function theorem. Also, the formula for $B^{N^{\prime}}\left(q_{-i}\right)$ is obtained 
using the implicit function theorem. The numerator in this formula is the derivative of firm $i$ 's marginal revenue with respect to $q_{-i}$. The denominator is the negative of the derivative of the firm's marginal profit with respect to its own quantity choice $q_{i}$. The ratio is negative because the numerator is negative and the denominator is positive. Next we consider the twofold iterate of $B^{N}$.

Lemma 5. For all $N \in \mathbb{N}$ with $N \geq 2$ :

(i) If $B^{N}(0)>(N /(N-1)) D\left(C^{\prime}(0)\right)$, then there are $y^{N}, z^{N}>0$ with $y^{N}<z^{N}$ such that $\bar{B}^{N}\left(q_{-i}\right)=0$ if and only if $q_{-i} \in\left[0, y^{N}\right]$ and $\bar{B}^{N}\left(q_{-i}\right)=$ $B^{N}(0)$ if and only if $q_{-i} \geq z^{N}$. Moreover, on $\left[y^{N}, z^{N}\right] \tilde{B}^{N}$ is continuously differentiable. Its derivative satisfies $\tilde{B}^{N^{\prime}}\left(q_{-i}\right)=B^{N^{\prime}}\left(q_{-i}\right) B^{N^{\prime}}\left(B^{N}\left(q_{-i}\right)\right)(>0)$ for all $q_{-i} \in\left[y^{N}, z^{N}\right]$.

(ii) If $B^{N}(0) \leq(N /(N-1)) D\left(C^{\prime}(0)\right)$, then there is some $z^{N}>0$ such that $\tilde{B}^{N}\left(q_{-i}\right)=B^{N}(0)$ if and only if $q_{-i} \geq z^{N}$. Moreover, on $\left[0, z^{N}\right]$, $\bar{B}^{N}$ is continuously differentiable. Its derivative is given by $\tilde{B}^{N^{\prime}}\left(q_{-i}\right)=$ $B^{N^{\prime}}\left(q_{-i}\right)\left(B^{N^{\prime}}\left(B^{N}\left(q_{-i}\right)\right)(>0)\right.$ for all $q \in\left[0, z^{N}\right]$.

Lemma 5 is the analogue of Lemma 2 . We finally turn to the relevance of fixed points of $\bar{B}^{N}$ for the sets of strategies that survive iterated deletion of strongly dominated strategies:

Lemma 6. For all $N \in \mathbb{N}$ with $N \geq 2$ :

(i) $q^{N . C}$ is a fixed point of $\bar{B}^{N}$.

(ii) $\min R^{N}$ is the smallest fixed point of $\dot{B}^{N}$ and $\max R^{N}$ is the largest fixed point of $\bar{B}^{N}$.

Our next step is to describe first the relation between the function $F$ and the functions $B^{N}$, and then the relation between the function $F$ and the functions $\tilde{B}^{N}$, in both cases assuming that $N$ is large. Roughly speaking, what we shall show is that the sequence $\left\{B^{N}\right\}_{N \in \mathbb{N}}$ converges in the uniform $C^{1}$-topology to the function $F$ and, analogously, that the sequence $\left\{\tilde{B}^{N}\right\}_{N \in \mathbb{N}}$ converges in the uniform $C^{1}$-topology to the function $\tilde{F}$. The formal statements of these results are slightly more complicated because differentiability issues must be taken care of.

Lemma 7. (i) For $N \rightarrow \infty$ the sequence $\left\{B^{N}\right\}_{N \in \mathbb{N}}$ converges in the uniform topology to $F$.

(ii) Let $r \in\left(0, D\left(C^{\prime}(0)\right)\right.$. Then there exists some $\bar{N} \in \mathbb{N}$ such that for all $N \geq \bar{N} B^{N}$ is continuously differentiable on $[0, r]$, and, on this interval, the sequence of derivatives, $\left\{B^{N \prime}\right\}_{N \geq \bar{N}}$, converges uniformly to the derivative $F^{\prime}$. 
Proof. (i) We first show pointwise convergence. So consider some fixed $q_{-i} \in \mathbb{R}_{+}$, and suppose that the sequence $\left\{B^{N}\left(q_{-i}\right)\right\}_{N \in \mathbb{N}}$ does not converge to $F\left(q_{-i}\right)$. By possibly taking a subsequence we can achieve that the sequence does have a well-defined limit. Denote this limit by $\bar{F}\left(q_{-i}\right)$.

For large enough $N$ we have $B^{N}\left(q_{-i}\right) \neq F\left(q_{-i}\right)$, and hence, since $B^{N}\left(q_{-i}\right)$ is the unique best reply,

$$
\begin{aligned}
P\left(\frac{N-1}{N} q_{i}+\frac{1}{N} B^{N}\left(q_{-i}\right)\right) & B^{N}\left(q_{-i}\right)-C\left(B^{N}\left(q_{-i}\right)\right) \\
& >P\left(\frac{N-1}{N} q_{i}+\frac{1}{N} F\left(q_{-i}\right)\right) F\left(q_{-i}\right)-C\left(F\left(q_{-i}\right)\right) .
\end{aligned}
$$

Taking limits for $N \rightarrow \infty$, we obtain

$$
P\left(q_{-i}\right) \bar{F}\left(q_{-i}\right)-C\left(\bar{F}\left(q_{-i}\right)\right) \geq P\left(q_{-i}\right) F\left(q_{-i}\right)-C\left(F\left(q_{-i}\right)\right)
$$

which contradicts the fact that the quantity $F\left(q_{-i}\right)$ is the unique profit maximizing choice if the firm takes the price $P\left(q_{-i}\right)$ as given and fixed. Thus pointwise convergence is established.

Next, Exercise 13 in Rudin (1976, p. 167) shows that pointwise convergence implies uniform convergence if the functions $B^{N}$ are monotonically decreasing, if the function $F$ is monotonically decreasing and continuous, and if there is an interval such that all the functions $B^{N}$ and the function $F$ take values only in this interval. Lemmas 1 and 4 show that these conditions are satisfied, and hence uniform convergence follows.

(ii) The assertion about the differentiability of the functions $B^{N}$ on the interval $[0, r]$ follows immediately from part (i) of the current lemma and Lemmas 1 and 4. Uniform convergence of the derivatives follows then from inspection of the derivatives in Lemmas 1 and 4, using again part (i) of the current lemma. We omit the details.

Q.E.D.

Next, we state the analogue of Lemma 7 for the twofold iterates of the functions considered.

Lemma 8 . (i) For $N \rightarrow \infty$ the sequence $\left\{\tilde{B}^{N}\right\}_{N \in \mathbb{N}}$ converges in the uniform topology to $\bar{F}$.

(ii) Let $r^{1}, r^{2} \in(y, z)$ (in case (i) of Lemma 2), resp. $r^{1}, r^{2} \in(0, z)$ (in case (ii) of Lemma 2), satisfy $r^{1}<r^{2}$. Then there exists some $\bar{N} \in \mathbb{N}$ such that, for all $N \geq \bar{N}, \tilde{B}^{N}$ is continuously differentiable on $\left[r^{1}, r^{2}\right]$, and, on this interval, the sequence of derivatives $\left\{\tilde{B}^{N^{\prime}}\right\}_{N \geq \bar{N}}$ converges uniformly to the derivative $\bar{F}^{\prime}$. 
Lemma 8 follows from Lemma 7. We are now in a position to prove the proposition.

Proof of Part (i) of the Proposition. By Lemma 6 it suffices to prove that there is some $\bar{N} \in \mathbb{N}$ such that for all $N \geq \bar{N}$ the function $\bar{B}^{N}$ has a unique fixed point. To show this we observe first that the conditions in part (i) of the proposition are satisfied if and only if the function $\tilde{F}$ has the unique fixed point $q^{w}$ and if, moreover, $F^{\prime}\left(q^{w}\right)<1$. This is an implication of Lemmas 1-3.

Now denote by $I$ some closed, finite interval such that $q^{W}$ is contained in the interior of $I$, such that $\bar{F}$ is continuously differentiable on $I$, and such that, for all $q \in I, \tilde{F}^{\prime}(q)<1$. The existence of such an interval follows from the fact that $\tilde{F}$ is continuously differentiable in the neighbourhood of $q^{W}$. Moreover, let $\varepsilon \equiv \min _{q \in I} 1-\tilde{F}^{\prime}(q)$ and let $\delta \cong \inf _{q \in \mathbb{R}_{+} \backslash l}|\bar{F}(q)-q|$. Observe that $0<\varepsilon<1$ and $\delta>0$.

Now, parts (i) and (ii) of Lemma 8 imply that we can find some $\bar{N} \in \mathbb{N}$ such that for all $N \geq \bar{N}:\left|\bar{B}^{N^{\prime}}(q)-\bar{F}^{\prime}(q)\right|<\varepsilon$ for all $q \in I$ and $\left|\bar{B}^{N}(q)-\tilde{F}(q)\right|<\delta$ for all $q \in \mathbb{R}_{+} \backslash$ I. Consider some given and fixed $N$ with $N \geq \bar{N}$. By Lemma $6, \bar{B}^{N}$ has at least one fixed point. The second of the previous inequalities implies that for $N \geq \bar{N}: \bar{B}^{N}(q) \neq q$ for all $q \in \mathbb{R}_{+} \backslash I$; i.e., $\bar{B}^{N}$ cannot have a fixed point outside of $I$. Hence it suffices to show that $\tilde{B}^{N}$ cannot have two fixed points that are contained in $I$. But if it had, then, by the mean value theorem, there would have to be a $q$ in between the two fixed points such that $\bar{B}^{N^{\prime}}(q)=1$. But this would contra dict the first of the two inequalities above.

Q.E.D.

Proof of Part (ii) of the Proposition. Let $q^{1}, q^{2}, q^{3}, \ldots$ be a cobweb sequence, and suppose that $q^{1}<q^{3}$. Observe that then $q^{1}>q^{W}>q^{2}$ must be true. Let $\hat{q} \in\left(q^{2}, q^{W}\right)$. Since $F$ is continuous $F(\hat{q})$ will be close to $F\left(q^{2}\right)=q^{3}$ if $\hat{q}$ is close to $q^{2}$. Hence, for $\hat{q}$ sufficiently close to $q^{2}$ we will have $F(\hat{q})>q^{1}$. In the following we shall assume that $\hat{q}$ is chosen so that this inequality holds.

Consider the interval $\left[\hat{q}, q^{\prime}\right]$. The monotonicity and continuity of the function $F$ implies that the set $\left\{q \in \mathbb{B}_{+} \mid q=F(r)\right.$ for some $\left.r \in\left[\hat{q}, q^{\prime}\right]\right\}$ will be an interval, the upper boundary of which is $F(\hat{q})$, which is by the above construction strictly greater than $q^{\prime}$, and the lower boundary of which is $F\left(q^{1}\right)$ which is equal to $q^{2}$, and which is hence strictly smaller than $\hat{q}$.

Lemma 7 , together with the monotonicity and continuity of the functions $B^{N}$, implies then that there is some $\bar{N} \in \mathbb{N}$ such that for all $N \geq \bar{N}$ also the set $\left\{q \in \mathbb{R}_{+} \mid q=B^{N}\left(q_{-i}\right)\right.$ for some $\left.q_{-i} \in\left[\hat{q}, q^{\prime}\right]\right\}$ will be an interval, the lower boundary of which is strictly smaller than $\hat{q}$ and the upper boundary of which is strictly greater than $q^{1}$. 
Hence for $N \geq \bar{N}:\left\{q \in \mathbb{R}_{+} \mid q=B^{N}(r)\right.$ for some $\left.r \in\left[\hat{q}, q^{1}\right]\right\} \subseteq\left[\hat{q}, q^{1}\right]$. This implies that, if $N \geq \bar{N}$, at no stage of the procedure of iterated deletion of strongly dominated strategies can any element of $\left[\hat{q}, q^{l}\right]$ be eliminated. Hence $\left[\hat{q}, q^{1}\right] \subseteq R^{N}$ for all $N \geq \bar{N}$. Moreover, by construction, $q^{W}$ is in the interior of $\left[\hat{q}, q^{1}\right]$. Thus the claim is established. Q.E.D.

\section{REFERENCES}

Allen, B., AND Hellwig, M. (1986). "Bertrand-Edgeworth Oligopoly in Large Markets," Rev. Econ. Stud. 53, 175-204.

Al-Nowaihi, A., AND Levine, P. L. (1985). "The Stability of the Cournot Oligopoly Model: A Re-assessment," J. Econ. Theory 35, 307-321.

BASu, K. (1991). "A Characterization of the Class of Rationalizable Equilibria of Oligopoly Gr .es," mimeograph. Princeton, NJ: Princeton University.

Bernhfim, B. (1984). "Rationalizable Strategic Behavior," Econometrica 52, 10071028.

BÖRGERS, T. (1992). "Iterated Elimination of Dominated Strategies in a Bertrand-Edgeworth Model," Rev. Econ. Stud. 59, 163-176.

Fisher, F. M. (1961). "The Stability of the Cournot Oligopoly Solution: The Effects of Speeds of Adjustment and Increasing Marginal Costs," Rev. Econ. Stud. 28, 125-135.

Friedman, J. (1982). "Oligopoly Theory," in Handbook of Mathematical Economics (K. J. Arrow and M. D. Intriligator, Eds.), Vol. II, pp. 491-534. Amsterdam: NorthHolland.

GUESNERIE, R. (1992). "An Exploration of the Eductive Justifications of the Rational Expectations Hypothesis," Amer. Econ. Rev. 82, 1254-1278.

HaHn, F. (1962), "The Stability of the Cournot Oligopoly Solution," Rev. Econ. Stud. 29, 329-331.

Janssen, M. C. W. (1993). "Microfoundations; A Critical Inquiry," London: Routledge.

McManus, M., And Quandt, R. E. (1961). "Comments on the Stability of the Cournot Oligopoly Model," Rev. Econ. Stud. 28, 136-139.

Milgrom, P., and Roberts, J. (1990). "Rationalizability, Learning, and Equilibrium in Games with Strategic Complementarities," Econometrica 58, 1255-1277.

Milgrom, P., and Roberts, J. (1991). "Adaptive and Sophisticated Learning in Normal Form Games," Games Econ. Behav. 3, 82-100.

Moulin, H. (1984). "Dominance Solvability and Cournot Stability," Math. Soc. Sci. 7, 83-102.

NovsheK, W. (1985). "Perfectly Competitive Markets as the Limits of Cournot Markets," J. Econ. Theory 35, 72-82.

Rudin, W. (1976). Principles of Mathematical Analysis, 3rd ed. New York: McGraw-Hill.

Ruffin, R. J. (1971). "Cournot Oligopoly and Competitive Behaviour," Rev. Econ. Stud. 38, 493-502.

Theocharis, R. O. (1960). "On the Stability of the Cournot Solution of the Oligopoly Problem," Rev. Econ. Stud. 27, 133-134. 
Thorlund-Petersen, L. (1990). "Iterative Computation of Cournot Equilibrium," Games Econ. Behav. 2, 61-75.

VIVEs, X. (1986). "Rationing Rules and Bertrand-Edgeworth Equilibria in Large Markets," Econ. Lett. 21, 113-116.

VIVES, X. (1990). "Nash Equilibrium and Strategic Complements," J. Math. Econ. 19, 305-321. 